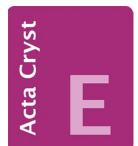

CRYSTALLOGRAPHIC COMMUNICATIONS

ISSN 2056-9890

Received 14 December 2017

Accepted 19 December 2017

Edited by A. J. Lough, University of Toronto, Canada

₹ Deceased July 7, 2015.

Keywords: crystal structure; nitrile; isocyanide; $\mathrm{N}$...I contacts; $\mathrm{C} . . \mathrm{I}$ contacts.

CCDC references: $1580006 ; 1581218$

Supporting information: this article has supporting information at journals.iucr.org/e

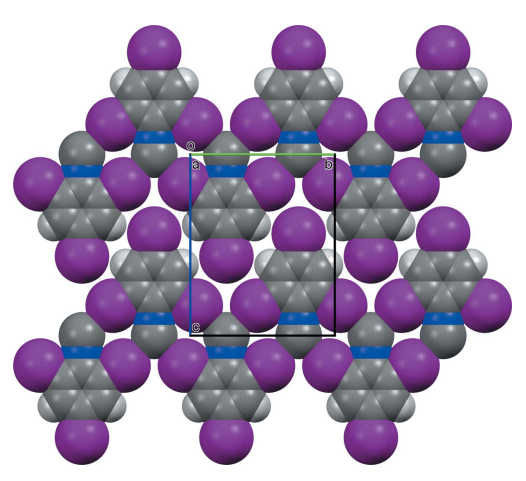

OPEN $\odot$ ACCESS

\section{Crystal structures of 2,4,6-triiodobenzonitrile and 2,4,6-triiodophenyl isocyanide}

\author{
Wayland E. Noland,* Doyle Britton, $\neq$ Gregory K. Sutton, Andrew K. Schneerer and \\ Kenneth J. Tritch
}

Department of Chemistry, University of Minnesota, 207 Pleasant St SE, Minneapolis, MN 55455, USA. *Correspondence
e-mail: nolan001@umn.edu

The title crystals, $\mathrm{C}_{7} \mathrm{H}_{2} \mathrm{I}_{3} \mathrm{~N}$, are isomorphous. Both molecules lie across two crystallographic mirror planes and a twofold axis. The principal supramolecular interaction is centric $R_{2}^{2}(10) \mathrm{CN} / \mathrm{NC} \cdot \mathrm{I}$ short contacts involving both ortho I atoms, with two contacts bisecting each cyano and isocyano group. These form ribbons along [010] and give rise to a planar sheet structure parallel to (100). All pairs of adjacent sheets have centric stacking, a mode not previously reported for sheets of this type. This study completes the series of homo-2,4,6trihalobenzonitriles, in which I atoms give the strongest $\mathrm{CN} \cdots X$ and $\mathrm{NC} \cdots X$ interactions $(X=\mathrm{F}, \mathrm{Cl}, \mathrm{Br}, \mathrm{I})$.

\section{Chemical context}

The strength of cyano-halo interactions tends to increase with increasing polarizability, or the elemental period, of the halogen. Structure-directing $\mathrm{CN} \cdots \mathrm{F}$ interactions are usually not observed (Bond et al., 2001). In crystals of the other 4-halobenzonitriles $(X=\mathrm{Cl}, \mathrm{Br}, \mathrm{I})$, parallel or antiparallel $C_{1}^{1}(7) \mathrm{CN} \cdots X$ chains dominate the secondary structures (Fig. 1; Desiraju \& Harlow, 1989). When the halo atom is moved to the 2-position, $R_{2}^{2}(10) \mathrm{CN} \cdots X$ rings can form, usually as inversion dimers. Halogenation at both ortho positions allows the formation of $\mathrm{CN} \cdots X$-derived ribbons or sheets. The aforementioned periodic trend is exhibited by the homo-2,4,6-trihalobenzonitriles. No $\mathrm{CN} \cdots \mathrm{F}$ contacts are observed in 2,4,6-trifluorobenzonitrile (F3CN). Instead, each $\mathrm{CN}$ group is bisected by two $\mathrm{CN} \cdot \cdot \mathrm{H}$ contacts (Fig. $2 a$; Britton, 2008). In 2,4,6-trichlorobenzonitrile $(\mathrm{Cl} 3 \mathrm{CN})$, half of these have been replaced by $\mathrm{CN}$. Cl contacts (Fig. $2 b$; Pink et al., 2000). In 2,4,6-tribromobenzonitrile ( $\mathrm{Br} 3 \mathrm{CN})$, no $\mathrm{CN} \cdots \mathrm{H}$ contacts are found, and each $\mathrm{CN}$ group is bisected by two CN...Br contacts (Fig. 2c; Britton et al., 2016).

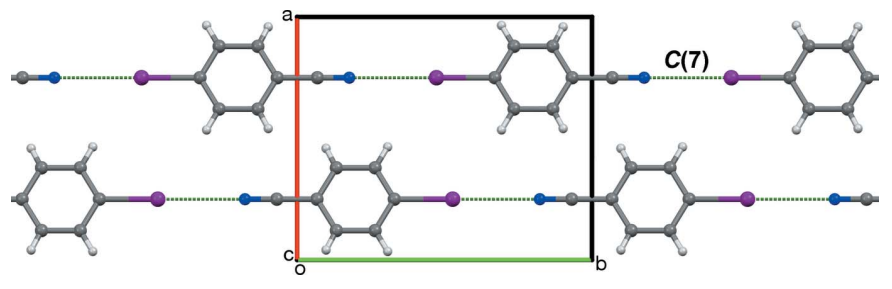

Figure 1

Several molecules in the crystal of 4-iodobenzonitrile (4ICN), viewed along [001]. Dashed green lines represent CN … I short contacts, which collectively form a $C(7)$ chain motif along [010]. All previously reported 4-iodobenzonitriles form similar chains. 


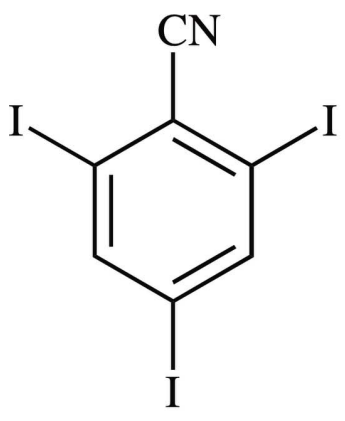

$\mathrm{I} 3 \mathrm{CN}$

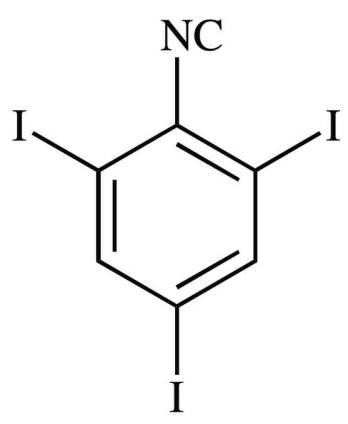

$\mathrm{I} 3 \mathrm{NC}$

\section{Database survey}

No entries were found in the most recent update of the Cambridge Structural Database (Version 5.37, May 2017; Groom et al., 2016) that have I atoms at both ortho positions of a benzonitrile. Four of the five crystalline 2-iodobenzonitriles have CN ...I contacts (Britton, 2001, 2004; Ketels et al., 2017; Lam \& Britton, 1974); the fifth is a cyano alcohol that forms $\mathrm{O}-\mathrm{H} \cdots \mathrm{NC}$ hydrogen bonds (Salvati et al., 2008). The 3-iodo analogs do not pack as efficiently. Three of the four examples feature I - I I contacts (Britton, 2006; Merz, 2006); packing in the fourth example is directed by hydrogen bonding between acetamido groups (Garden et al., 2007). All five reported 4-iodobenzonitriles form $C(7) \mathrm{CN} \cdots \mathrm{I}$ chains (Fig. 1; Bond et al., 2001; Britton, 2004; Desiraju \& Harlow, 1989; Gleason \& Britton, 1978). It is pertinent to determine the crystal structure of 2,4,6-triiodobenzonitrile ( $\mathrm{I} 3 \mathrm{CN})$ to complete the series of homo-2,4,6-trihalobenzonitriles, and to determine whether the primary packing interaction is $\mathrm{CN} \cdots \mathrm{I}$-derived $C(7)$ chains, $R_{2}^{2}(10)$ rings, or another motif. 2,4,6-Triiodophenyl isocyanide (I3NC) is included to contribute to the library of corresponding halogenated nitrile-isocyanide crystal pairs. (a)
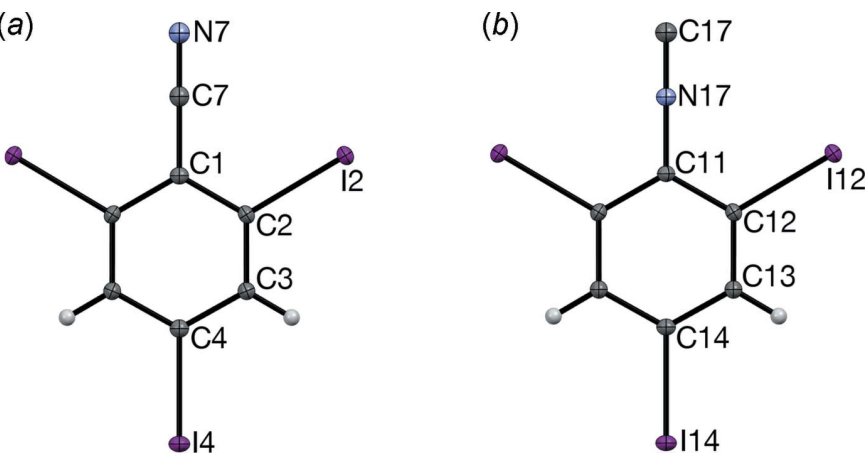

Figure 3

The molecular structures of $(a) \mathrm{I} 3 \mathrm{CN}$ and $(b) \mathrm{I} 3 \mathrm{NC}$, with atom labeling and displacement ellipsoids at the $50 \%$ probability level. Unlabeled atoms are generated by the symmetry operation $\left(1-x, \frac{3}{2}-y, z\right)$.

\section{Structural commentary}

Molecules of I3CN and I3NC (Fig. 3) lie about a twofold axis and two orthogonal vertical mirror planes. Thus, they have crystallographically-imposed $C_{2 v}$ symmetry and are planar, with the para I atom (I4; I14) collinear with the $\mathrm{CN}$ and $\mathrm{NC}$ groups. All of the aryl bond angles are roughly $120^{\circ}$. The ortho I atoms (I2, I2'; I12, I12') are scissored slightly toward the ipso $\mathrm{C}$ atom $(\mathrm{C} 1$; $\mathrm{C} 11)$, which is probably caused by the intermolecular $\mathrm{CN} \cdots \mathrm{I}$ and $\mathrm{NC} \cdots \mathrm{I}$ short contacts. The bond lengths are typical for their respective functional groups.

\section{Supramolecular features}

Crystals of I3CN and I3NC are isomorphous. The $\mathrm{CN}$ and $\mathrm{NC}$ groups are bisected by $\mathrm{C} 7 \equiv \mathrm{N} 7 \cdots \mathrm{I} 2$ and $\mathrm{N} 17 \equiv \mathrm{C} 17 \cdots \mathrm{I} 12$ contacts (Table 1 ), forming ribbons of $R_{2}^{2}(10)$ rings parallel to (100) along [010]. Adjacent ribbons translate along [001]. The resulting planar sheet structure (Fig. 4) matches that observed in $\mathrm{Br} 3 \mathrm{CN}$ and the corresponding isocyanide (Br3NC) (Britton et al., 2016), and the 4-chloro (Britton, 2005) and 4-nitro (a)

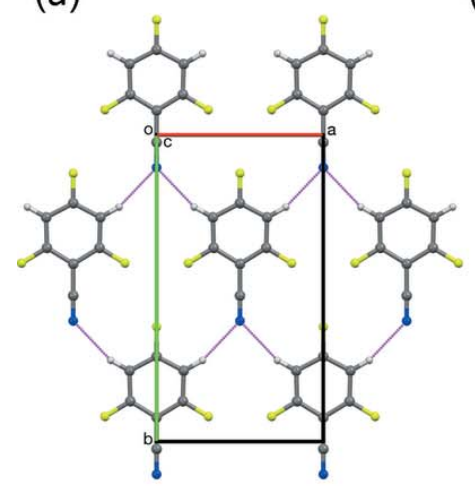

(b)

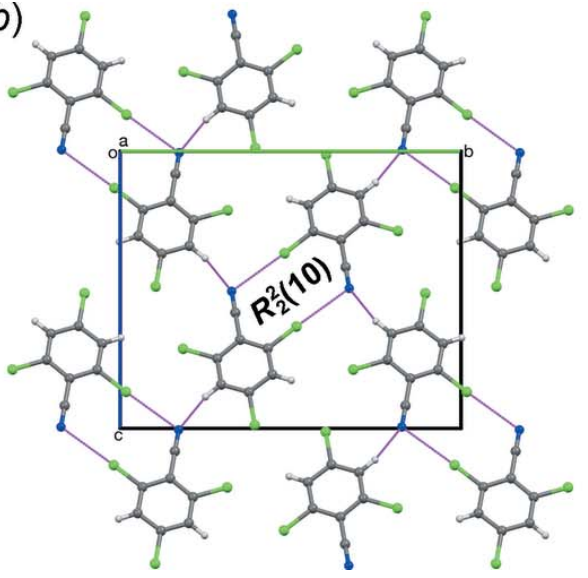

(c)

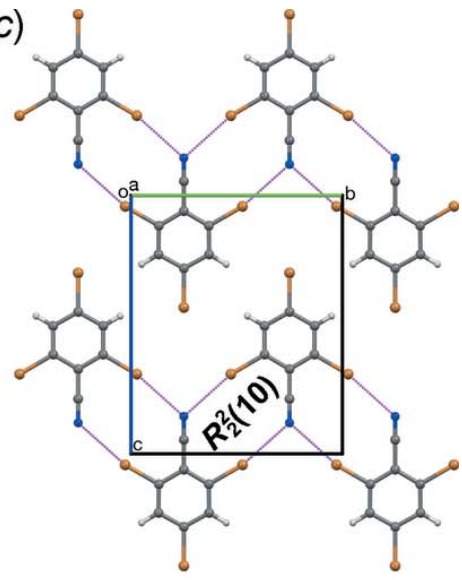

Figure 2

(a) A sheet in a crystal of $\mathrm{F} 3 \mathrm{CN}$, showing two $\mathrm{CN} \cdots \mathrm{H}$ contacts per $\mathrm{CN}$ group, viewed along [001]; $(b)$ A sheet in a crystal of $\mathrm{Cl} 3 \mathrm{CN}$, showing one $\mathrm{CN} \cdots \mathrm{H}$ and one $\mathrm{CN} \cdots \mathrm{Cl}$ contact per $\mathrm{CN}$ group, viewed along [100]; (c) A sheet in the $Z=8$ polytype of $\mathrm{Br} 3 \mathrm{CN}$, showing two $\mathrm{CN} \cdots \mathrm{Br}$ contacts per $\mathrm{CN}$ group, viewed along [100]. Dashed magenta lines represent short contacts. 
Table 1

Contact geometry $\left(\AA,^{\circ}\right)$ for I3CN and I3NC.

\begin{tabular}{llll}
\hline$A \equiv B \cdots \mathrm{I}$ & $A \equiv B$ & $B \cdots \mathrm{I}$ & $A \equiv B \cdots \mathrm{I}$ \\
\hline $\mathrm{C} 7 \equiv \mathrm{N} 7 \cdots \mathrm{I}^{\mathrm{i}}$ & $1.151(3)$ & $3.074(2)$ & $132.85(3)$ \\
$\mathrm{N} 17 \equiv \mathrm{C} 17 \cdots \mathrm{I} 12^{\mathrm{i}}$ & $1.164(3)$ & $3.106(2)$ & $134.18(3)$ \\
\hline
\end{tabular}

Symmetry code: (i) $-x+1, y-\frac{1}{2},-z+2$

(Noland \& Tritch, 2017) analogs of Br3CN. In crystals of I3CN and $\mathrm{I} 3 \mathrm{NC}$, all pairs of adjacent sheets have centric stacking along [100] (Fig. 5), with molecules stacked about a glide plane and an inversion center. In the polytypes of $\mathrm{Br} 3 \mathrm{CN}$ and $\mathrm{Br} 3 \mathrm{NC}$, adjacent sheets had combinations of centric and translational stacking, but not solely centric stacking. The 4-chloro analog had translational stacking. The 4-nitro analog had glide stacking, with no inversion center between stacked molecules. Thus, the all-centric stacking of I3CN and I3NC can be regarded as a new polytype in this series.

The mean $\mathrm{CN} \cdots X$ contact lengths can be compared for $X=$ $\mathrm{Cl}, \mathrm{Br}$, and I (Table 2). For 4-chlorobenzonitrile (4ClCN), 4-bromobenzonitrile (4BrCN), and 4-iodobenzonitrile (4ICN) (Table 2, col. 2), the contact distance decreases with increasing halogen size, highlighting the increase in contact strength (Desiraju \& Harlow, 1989). This trend is essentially mirrored among 2,4,6-trihalobenzonitriles (Table 2, col. 3), although the contact distance in $\mathrm{I} 3 \mathrm{CN}$ is $0.01 \AA$ larger than in $\mathrm{Br} 3 \mathrm{CN}$. The $\mathrm{N}$. . X non-bonded contact radii are listed (Table 2, col. 4; Rowland \& Taylor, 1996). The 'shortness' of contacts in 2,4,6trihalobenzonitriles is expressed as the ratios of contact radii to the respective contact distances (Table 2, col. 5). A similar comparison of $\mathrm{NC} \cdots X$ contact lengths in the corresponding trihalo isocyanides also shows decreasing contact length with increasing halogen size (Table 3 , col. 2 ). The NC $\cdots X$ contacts

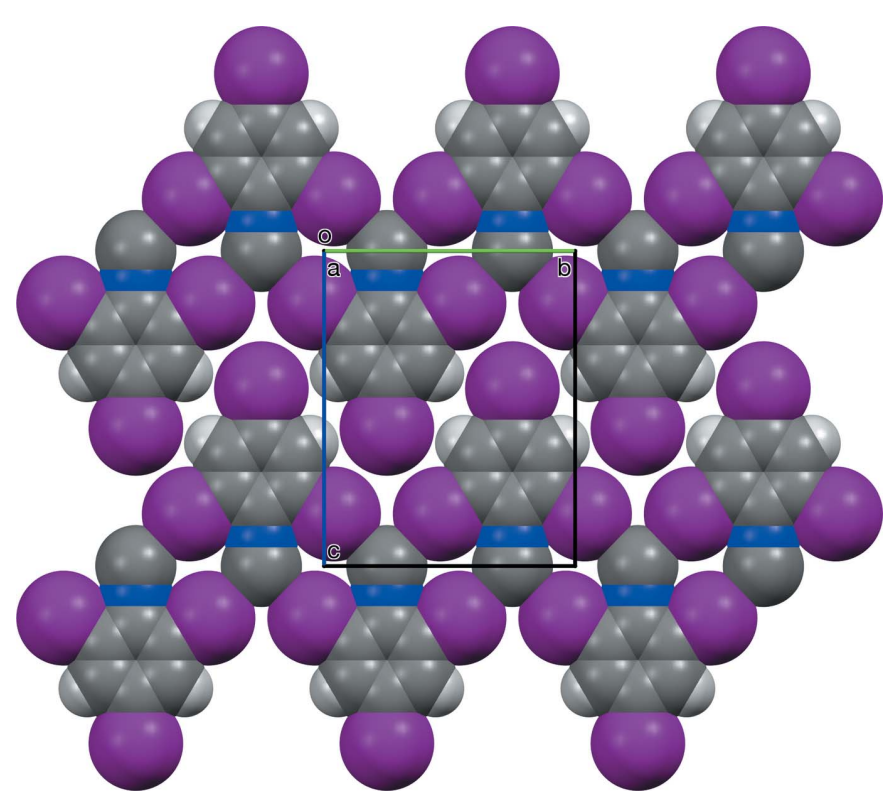

Figure 4

A space-filling drawing of the sheet structure of I3NC, viewed along [100].
Table 2

Mean $\mathrm{CN} \cdots X$ contact lengths $(\AA)$ in 4 -halobenzonitriles $(4 X \mathrm{CN})$ and 2,4,6-trihalobenzonitriles $(X 3 \mathrm{CN})$.

\begin{tabular}{lllll}
\hline$X$ & $4 X \mathrm{CN}$ & $X 3 \mathrm{CN}$ & $r[\mathrm{~N}+X](\AA)$ & {$[r / X 3 \mathrm{CN}]$} \\
\hline $\mathrm{Cl}$ & $3.370(4)$ & $3.153(2)$ & 3.35 & 1.06 \\
$\mathrm{Br}$ & $3.249(5)$ & $3.064(4)$ & 3.46 & 1.13 \\
$\mathrm{I}$ & $3.127(4)$ & $3.074(2)$ & 3.61 & 1.17 \\
\hline
\end{tabular}

Table 3

Mean NC $\cdots X$ contact lengths $(\AA)$ in 2,4,6-trihalophenyl isocyanides $(X 3 \mathrm{NC})$.

\begin{tabular}{llll}
\hline$X$ & $X 3 \mathrm{NC}$ & $r[\mathrm{C}+X]$ & {$[r / X 3 \mathrm{NC}]$} \\
\hline $\mathrm{Cl}$ & $3.245(3)$ & 3.49 & 1.08 \\
$\mathrm{Br}$ & $3.151(4)$ & 3.60 & 1.14 \\
$\mathrm{I}$ & $3.106(2)$ & 3.75 & 1.21 \\
\hline
\end{tabular}

have slightly greater shortness (Table 3, col. 4) than the corresponding $\mathrm{CN} \cdots X$ contacts. The $\mathrm{N} 17 \equiv \mathrm{C} 17 \cdots \mathrm{I} 12$ contacts in $\mathrm{I} 3 \mathrm{NC}$ are the strongest cyano/isocyano-halo interactions in this series.

\section{Synthesis and crystallization}

2,4,6-Triiodoaniline (I3NH2), adapted from the work of Jackson \& Whitmore (1915): Aniline $(1.0 \mathrm{~mL})$ and hydrochloric acid $(0.7 \mathrm{M}, 850 \mathrm{~mL})$ were combined and stirred in a round-bottomed flask. Iodine monochloride (8.2 g) was placed in a separate flask and then warmed to $315 \mathrm{~K}$. The two flasks were connected with a glass bridge. A slow stream of nitrogen was passed through the headspace in the second vessel so that the iodine monochloride was gradually swept into the first

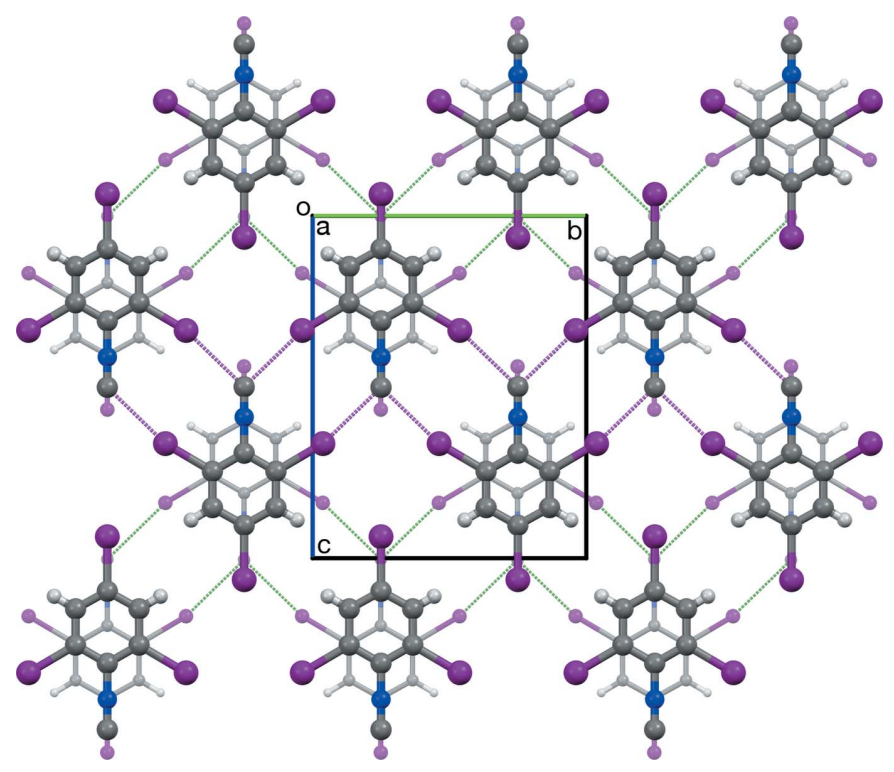

Figure 5

Two adjacent sheets in I3NC, viewed along [100], illustrating the centric stacking mode. Dashed magenta lines represent short contacts in the front layer. Molecules in the rear layer are drawn with smaller balls and sticks, lower opacity, and green dashed lines representing short contacts. 
Table 4

Experimental details.

\begin{tabular}{|c|c|c|}
\hline & $\mathrm{I} 3 \mathrm{CN}$ & $\mathrm{I} 3 \mathrm{NC}$ \\
\hline \multicolumn{3}{|l|}{ Crystal data } \\
\hline Chemical formula & $\mathrm{C}_{7} \mathrm{H}_{2} \mathrm{I}_{3} \mathrm{~N}$ & $\mathrm{C}_{7} \mathrm{H}_{2} \mathrm{I}_{3} \mathrm{~N}$ \\
\hline$M_{\mathrm{r}}$ & 480.80 & 480.80 \\
\hline Temperature (K) & 100 & 100 \\
\hline$a, b, c(\AA)$ & $7.0593(4), 10.5346(5), 13.0658(6)$ & 7.0552 (3), 10.4947 (5), $13.1557(5)$ \\
\hline$V\left(\AA^{3}\right)$ & $971.66(8)$ & $974.08(7)$ \\
\hline$Z$ & 4 & 4 \\
\hline Crystal size (mm) & $0.12 \times 0.10 \times 0.10$ & $0.15 \times 0.09 \times 0.07$ \\
\hline \multicolumn{3}{|l|}{ Data collection } \\
\hline Diffractometer & Bruker VENTURE PHOTON-II & Bruker VENTURE PHOTON-II \\
\hline Absorption correction & Multi-scan (SADABS; Sheldrick, 1996) & Multi-scan (SADABS; Sheldrick, 1996) \\
\hline$T_{\min }, T_{\max }$ & $0.281,0.344$ & $0.251,0.344$ \\
\hline $\begin{array}{l}\text { No. of measured, independent and } \\
\text { observed }[I>2 \sigma(I)] \text { reflections }\end{array}$ & $8436,1303,1261$ & $13040,1314,1267$ \\
\hline \multicolumn{3}{|l|}{ Refinement } \\
\hline$R\left[F^{2}>2 \sigma\left(F^{2}\right)\right], w R\left(F^{2}\right), S$ & $0.013,0.030,1.18$ & $0.011,0.024,1.14$ \\
\hline No. of reflections & 1303 & 1314 \\
\hline No. of parameters & 40 & 40 \\
\hline $\mathrm{H}$-atom treatment & H-atom parameters constrained & $\mathrm{H}$-atom parameters constrained \\
\hline$\Delta \rho_{\max }, \Delta \rho_{\min }\left(\mathrm{e} \AA^{-3}\right)$ & $0.89,-0.60$ & $0.72,-0.48$ \\
\hline
\end{tabular}

Computer programs: APEX3 (Bruker, 2012), SAINT (Bruker, 2012), SHELXT2014 (Sheldrick, 2015a), SHELXL2014 (Sheldrick, 2015b), Mercury (Macrae et al., 2008), publCIF (Westrip, 2010).

vessel over 2-4 d. After the transfer was complete, the reaction mixture was neutralized with $\mathrm{NaHCO}_{3}$ solution, followed by reduction of excess iodine by washing with $\mathrm{Na}_{2} \mathrm{~S}_{2} \mathrm{O}_{3}$ solution. Dichloromethane (approx. $100 \mathrm{~mL}$ ) was added, with stirring, until nearly all solids had dissolved. The organic portion was filtered through silica gel $(3 \mathrm{~cm} \mathrm{H} \times 4 \mathrm{~cm} \mathrm{D})$, and then the filter was washed with dichloromethane $(3 \times 20 \mathrm{~mL})$. The filtrate was placed in a loosely-covered beaker. After most of the dichloromethane had evaporated, beige needles were collected by suction filtration (4.48 g, 89\%). M.p. 459-460 K (lit. 459); ${ }^{1} \mathrm{H}$ NMR (300 MHz, $\left.\mathrm{CDCl}_{3}\right) \delta 7.864(s, 2 \mathrm{H}), 4.658(s$, $2 \mathrm{H}) ;{ }^{13} \mathrm{C} \mathrm{NMR}\left(75 \mathrm{MHz},\left(\mathrm{CD}_{3}\right)_{2} \mathrm{SO}\right) \delta 147.0$ (1C), 145.4 (2C), 83.0 (2C), 78.8 (1C); IR (KBr, cm ${ }^{-1}$ ) 3417, 3056, 2987, 1632, $1422,1265,741,704 ; \mathrm{MS}(\mathrm{EI}, \mathrm{m} / z)[\mathrm{M}]^{+}$calculated for $\mathrm{C}_{6} \mathrm{H}_{4} \mathrm{I}_{3} \mathrm{~N}$ 470.7472 , found 470.7470 .

2,4,6-Triiodobenzonitrile (I3CN), was prepared from I3NH2 (101 mg; Fig. 6) based on the Sandmeyer procedure described by Britton et al. (2016). Ethyl acetate $(20 \mathrm{~mL})$, toluene-4-sulfonic acid monohydrate $(77 \mathrm{mg})$, and isoamyl nitrite $(60 \mu \mathrm{L})$ were used in place of water, acetic and sulfuric acids, and sodium nitrite, respectively. The desired chromatographic fraction $\left(R_{f}=0.44\right.$ in 4:1 hexane-ethyl acetate) was concentrated on a rotary evaporator, giving a beige powder (67 mg, 65\%). M.p. 517-518 K; ${ }^{1} \mathrm{H} \quad \mathrm{NMR} \quad(500 \mathrm{MHz}$, $\left.\left(\mathrm{CD}_{3}\right)_{2} \mathrm{SO}\right) \delta 8.431(\mathrm{~s}, 2 \mathrm{H}, \mathrm{H} 3) ;{ }^{13} \mathrm{C} \mathrm{NMR}\left(126 \mathrm{MHz}, \mathrm{CD}_{2} \mathrm{Cl}_{2}\right) \delta$ 147.5 (2C, C3), 127.3 (1C, C1), 120.7 (1C, C7), 101.1 (1C, C4), 99.6 (2C, C2); IR ( NaCl, cm ${ }^{-1}$ ) 3070, 2227, 1532, 1359, 1206, 1081, 861, 787, 706; MS (ESI, $m / z)[M+\mathrm{Na}]^{+}$calculated for $\mathrm{C}_{7} \mathrm{H}_{2} \mathrm{I}_{3} \mathrm{~N}$ 503.7213, found 503.7216.
2,4,6-Triiodoformanilide (I3FA) was prepared from I3NH2 $(1.01 \mathrm{~g})$ according to the formylation procedure described by Britton et al. (2016), with 1,2-dichloroethane (10 mL and<smiles>Nc1c(I)cc(I)cc1I</smiles>

I3NH2
1. toluene-4-sulfonic acid, ethyl acetate

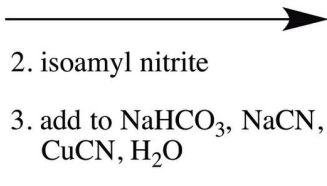

$65 \%$<smiles>N#Cc1c(I)cc(I)cc1I</smiles>

$\mathrm{I} 3 \mathrm{CN}$

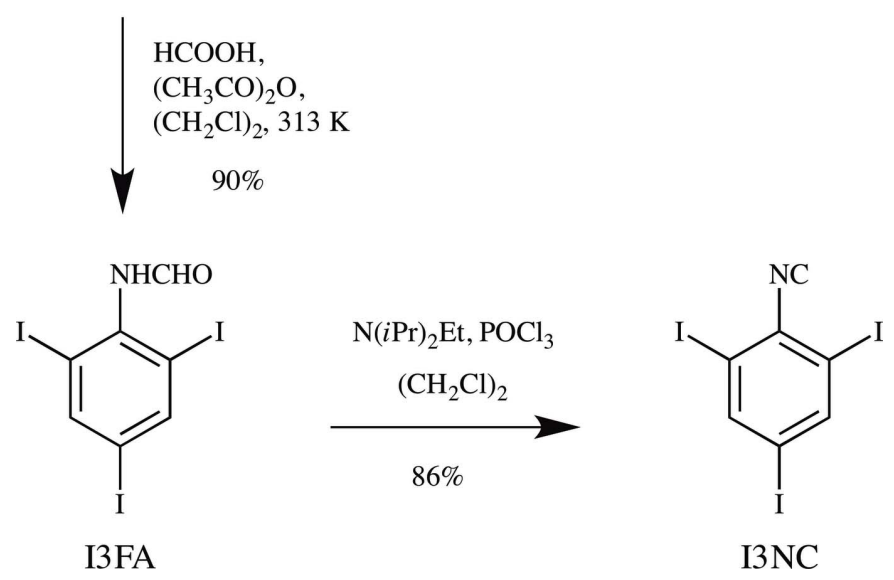

Figure 6

The synthesis of I3CN and I3NC. 
$100 \mathrm{~mL}$ ) in place of tetrahydrofuran, giving white needles (962 mg, 90\%). M.p. 557-558 K; ${ }^{1} \mathrm{H} \quad \mathrm{NMR} \quad(300 \mathrm{MHz}$, $\left(\mathrm{CD}_{3}\right)_{2} \mathrm{SO} ; 2$ conformers observed) $\delta 10.089$ ( $s, 1 \mathrm{H}$; major), 9.655 ( $s, 1 \mathrm{H}$; minor), 8.303 ( $s, 1 \mathrm{H}$; major), 8.278 ( $s, 2 \mathrm{H}$; minor), 8.233 ( $s, 2 \mathrm{H}$; major), 7.978 ( $s, 1 \mathrm{H}$; minor) $;{ }^{13} \mathrm{C} \mathrm{NMR}(126 \mathrm{MHz}$, $\left(\mathrm{CD}_{3}\right)_{2} \mathrm{SO} ; 2$ conformers observed) $\delta 164.4$ (1C; minor), 159.4 (1C; major), 146.4 (2C; minor), 146.1 (2C; major), 141.0 (1C; major), 140.4 (1C; minor), 102.4 (2C; minor), 101.9 (2C; major), 95.9 (1C; minor), 95.6 (1C; major); IR ( $\left.\mathrm{NaCl}, \mathrm{cm}^{-1}\right)$ 3221, 3076, 2919, 1637, 1490, 1380, 1232, 1143, 857, 794, 703, 682; MS (ESI, $m / z)[\mathrm{M}-\mathrm{H}]^{-}$calculated for $\mathrm{C}_{7} \mathrm{H}_{4} \mathrm{I}_{3} \mathrm{NO}$ 497.7354, found 497.7365 .

2,4,6-Triiodophenyl isocyanide (I3NC) was prepared from I3FA (397 mg) according to the dehydration procedure described by Britton et al. (2016), giving a white powder (330 mg, 86\%). M.p. 467-468 K; ${ }^{1} \mathrm{H}$ NMR (300 MHz, $\mathrm{CDCl}_{3}$ ) $8.198(s, 2 \mathrm{H}, \mathrm{H} 13) ;{ }^{13} \mathrm{C} \mathrm{NMR}\left(126 \mathrm{MHz},\left(\mathrm{CD}_{3}\right)_{2} \mathrm{SO}\right) \delta 170.0$ (1C, C17), 146.2 (2C, C13), 133.8 (1C, C11), 98.8 (1C, C14), 97.7 (2C, C12); IR (KBr, cm ${ }^{-1}$ ) 3073, 3037, 2920, 2126, 1529, 1079, 861, 704; MS (ESI, $m / z)[M-\mathrm{H}]^{-}$calculated for $\mathrm{C}_{7} \mathrm{H}_{2} \mathrm{I}_{3} \mathrm{~N}$ 479.7249 , found 479.7226 .

Crystallization: Crystals of $\mathrm{I} 3 \mathrm{CN}$ and I3NC were prepared by slow evaporation of acetonitrile solutions at ambient temperature, followed by decantation and then washing with pentane.

\section{Refinement}

Crystal data, data collection and structure refinement details are summarized in Table 4. A direct-methods solution was calculated, followed by full-matrix least squares/differenceFourier cycles. All $\mathrm{H}$ atoms were placed in calculated positions $(\mathrm{C}-\mathrm{H}=0.95 \AA)$ and refined as riding atoms with $U_{\text {iso }}(\mathrm{H})$ set to $1.2 U_{\text {eq }}(\mathrm{C})$.

\section{Acknowledgements}

The authors thank Victor G. Young, Jr. (X-Ray Crystallographic Laboratory, University of Minnesota) for assistance with the crystallographic determination, and the Wayland E. Noland Research Fellowship Fund at the University of Minnesota Foundation for generous financial support of this project. This work was taken in large part from the $\mathrm{PhD}$ thesis of KJT (Tritch, 2017).

\section{References}

Bond, A. D., Davies, J. E., Griffiths, J. \& Rawson, J. M. (2001). Acta Cryst. E57, o231-o233.

Britton, D. (2001). Acta Cryst. E57, o702-o704.

Britton, D. (2004). Acta Cryst. E60, o184-o186.

Britton, D. (2005). Acta Cryst. E61, o1726-o1727.

Britton, D. (2006). Acta Cryst. B62, 109-117.

Britton, D. (2008). Acta Cryst. C64, o583-0585.

Britton, D., Noland, W. E. \& Tritch, K. J. (2016). Acta Cryst. E72, 178183.

Bruker (2012). APEX3 and SAINT. Bruker AXS, Inc., Madison, WI, USA.

Desiraju, G. R. \& Harlow, R. L. (1989). J. Am. Chem. Soc. 111, $6757-$ 6764.

Garden, S. J., Custódio, C. de A., Wardell, J. L., Low, J. N. \& Glidewell, C. (2007). Acta Cryst. C63, o408-o410.

Gleason, W. B. \& Britton, D. (1978). Cryst. Struct. Commun. 7, 365370.

Groom, C. R., Bruno, I. J., Lightfoot, M. P. \& Ward, S. C. (2016). Acta Cryst. B72, 171-179.

Jackson, C. L. \& Whitmore, F. C. (1915). J. Am. Chem. Soc. 37, 15221537.

Ketels, M., Konrad, D. B., Karaghiosoff, K., Trauner, D. \& Knochel, P. (2017). Org. Lett. 19, 1666-1669.

Lam, S. \& Britton, D. (1974). Acta Cryst. B30, 1119-1120.

Macrae, C. F., Bruno, I. J., Chisholm, J. A., Edgington, P. R., McCabe, P., Pidcock, E., Rodriguez-Monge, L., Taylor, R., van de Streek, J. \& Wood, P. A. (2008). J. Appl. Cryst. 41, 466-470.

Merz, K. (2006). Cryst. Growth Des. 6, 1615-1619.

Noland, W. E. \& Tritch, K. J. (2017). IUCrData, 2, x171617.

Pink, M., Britton, D., Noland, W. E. \& Pinnow, M. J. (2000). Acta Cryst. C56, 1271-1273.

Rowland, R. S. \& Taylor, R. (1996). J. Phys. Chem. 100, 7384-7391.

Salvati, M. E., Balog, A., Shan, W., Rampulla, R., Giese, S., Mitt, T., Furch, J. A., Vite, G. D., Attar, R. M., Jure-Kunkel, M., Geng, J., Rizzo, C. A., Gottardis, M. M., Krystek, S. R., Gougoutas, J., Galella, M. A., Obermeier, M., Fura, A. \& Chandrasena, G. (2008). Bioorg. Med. Chem. Lett. 18, 1910-1915.

Sheldrick, G. M. (1996). SADABS. University of Göttingen, Germany.

Sheldrick, G. M. (2015a). Acta Cryst. A71, 3-8.

Sheldrick, G. M. (2015b). Acta Cryst. C71, 3-8.

Tritch, K. J. (2017). PhD thesis, University of Minnesota, Minneapolis, MN, USA.

Westrip, S. P. (2010). J. Appl. Cryst. 43, 920-925. 


\section{supporting information}

Acta Cryst. (2018). E74, 98-102 [https://doi.org/10.1107/S2056989017018217]

\section{Crystal structures of 2,4,6-triiiodobenzonitrile and 2,4,6-triiodophenyl isocyanide}

\section{Wayland E. Noland, Doyle Britton, Gregory K. Sutton, Andrew K. Schneerer and Kenneth J.}

Tritch

\section{Computing details}

For both structures, data collection: APEX3 (Bruker, 2012); cell refinement: SAINT (Bruker, 2012); data reduction: SAINT (Bruker, 2012); program(s) used to solve structure: SHELXT2014 (Sheldrick, 2015a); program(s) used to refine structure: SHELXL2014 (Sheldrick, 2015b); molecular graphics: Mercury (Macrae et al., 2008); software used to prepare material for publication: publCIF (Westrip, 2010).

\section{2,4,6-Triiodobenzonitrile (I3CN)}

\section{Crystal data}

$\mathrm{C}_{7} \mathrm{H}_{2} \mathrm{I}_{3} \mathrm{~N}$

$M_{r}=480.80$

Orthorhombic, Imma

$a=7.0593(4) \AA$

$b=10.5346(5) \AA$

$c=13.0658(6) \AA$

$V=971.66(8) \AA^{3}$

$Z=4$

$F(000)=840$

\section{Data collection}

Bruker VENTURE PHOTON-II diffractometer

Radiation source: micro-focus $\varphi$ and $\omega$ scans

Absorption correction: multi-scan (SADABS; Sheldrick, 1996)

$T_{\min }=0.281, T_{\max }=0.344$

8436 measured reflections

\section{Refinement}

Refinement on $F^{2}$

Least-squares matrix: full

$R\left[F^{2}>2 \sigma\left(F^{2}\right)\right]=0.013$

$w R\left(F^{2}\right)=0.030$

$S=1.18$

1303 reflections

40 parameters

0 restraints
$D_{\mathrm{x}}=3.287 \mathrm{Mg} \mathrm{m}^{-3}$

Melting point: $517 \mathrm{~K}$

Mo $K \alpha$ radiation, $\lambda=0.71073 \AA$

Cell parameters from 2721 reflections

$\theta=2.5-36.4^{\circ}$

$\mu=9.59 \mathrm{~mm}^{-1}$

$T=100 \mathrm{~K}$

Square bipyramid, colorless

$0.12 \times 0.10 \times 0.10 \mathrm{~mm}$

1303 independent reflections

1261 reflections with $I>2 \sigma(I)$

$R_{\text {int }}=0.024$

$\theta_{\max }=36.4^{\circ}, \theta_{\min }=2.5^{\circ}$

$h=-9 \rightarrow 11$

$k=-17 \rightarrow 17$

$l=-21 \rightarrow 21$

Hydrogen site location: inferred from neighbouring sites

$\mathrm{H}$-atom parameters constrained

$w=1 /\left[\sigma^{2}\left(F_{\mathrm{o}}^{2}\right)+(0.0078 P)^{2}+1.2371 P\right]$

where $P=\left(F_{\mathrm{o}}{ }^{2}+2 F_{\mathrm{c}}{ }^{2}\right) / 3$

$(\Delta / \sigma)_{\max }=0.001$

$\Delta \rho_{\max }=0.89 \mathrm{e} \AA^{-3}$

$\Delta \rho_{\min }=-0.60$ e $\AA^{-3}$ 
Extinction correction: SHELXL2014

(Sheldrick, 2015b),

$\mathrm{Fc}^{*}=\mathrm{kFc}\left[1+0.001 \mathrm{xFc}^{2} \lambda^{3} / \sin (2 \theta)\right]^{-1 / 4}$

Extinction coefficient: $0.00199(9)$

\section{Special details}

Geometry. All esds (except the esd in the dihedral angle between two 1.s. planes) are estimated using the full covariance matrix. The cell esds are taken into account individually in the estimation of esds in distances, angles and torsion angles; correlations between esds in cell parameters are only used when they are defined by crystal symmetry. An approximate (isotropic) treatment of cell esds is used for estimating esds involving l.s. planes.

Fractional atomic coordinates and isotropic or equivalent isotropic displacement parameters $\left(\AA^{2}\right)$

\begin{tabular}{lllll}
\hline & $x$ & $y$ & $z$ & $U_{\text {iso }} * U_{\text {eq }}$ \\
\hline I2 & 0.5000 & $0.46395(2)$ & $0.83491(2)$ & $0.01267(4)$ \\
$\mathrm{I} 4$ & 0.5000 & 0.7500 & $0.43429(2)$ & $0.01359(4)$ \\
$\mathrm{N} 7$ & 0.5000 & 0.7500 & $1.00506(18)$ & $0.0167(4)$ \\
$\mathrm{C} 1$ & 0.5000 & 0.7500 & $0.80692(18)$ & $0.0103(4)$ \\
$\mathrm{C} 2$ & 0.5000 & $0.63456(15)$ & $0.75302(13)$ & $0.0105(3)$ \\
$\mathrm{C} 3$ & 0.5000 & $0.63428(16)$ & $0.64671(13)$ & $0.0117(3)$ \\
$\mathrm{H} 3$ & 0.5000 & 0.5565 & 0.6100 & $0.014^{*}$ \\
$\mathrm{C} 4$ & 0.5000 & 0.7500 & $0.59458(19)$ & $0.0113(4)$ \\
$\mathrm{C} 7$ & 0.5000 & 0.7500 & $0.9170(2)$ & $0.0130(4)$ \\
\hline
\end{tabular}

Atomic displacement parameters $\left(\AA^{2}\right)$

\begin{tabular}{lllllll}
\hline & $U^{11}$ & $U^{22}$ & $U^{33}$ & $U^{12}$ & $U^{13}$ & $U^{23}$ \\
\hline I2 & $0.01764(6)$ & $0.00914(5)$ & $0.01122(5)$ & 0.000 & 0.000 & $0.00198(3)$ \\
I4 & $0.01801(8)$ & $0.01463(7)$ & $0.00812(6)$ & 0.000 & 0.000 & 0.000 \\
N7 & $0.0218(11)$ & $0.0137(9)$ & $0.0145(9)$ & 0.000 & 0.000 & 0.000 \\
C1 & $0.0104(9)$ & $0.0113(9)$ & $0.0094(8)$ & 0.000 & 0.000 & 0.000 \\
C2 & $0.0123(6)$ & $0.0088(6)$ & $0.0104(6)$ & 0.000 & 0.000 & $0.0017(5)$ \\
C3 & $0.0157(7)$ & $0.0095(6)$ & $0.0099(6)$ & 0.000 & 0.000 & $0.0002(5)$ \\
C4 & $0.0121(9)$ & $0.0115(9)$ & $0.0104(9)$ & 0.000 & 0.000 & 0.000 \\
C7 & $0.0137(10)$ & $0.0120(9)$ & $0.0133(10)$ & 0.000 & 0.000 & 0.000 \\
\hline
\end{tabular}

Geometric parameters $\left(\AA,{ }^{o}\right)$

\begin{tabular}{llll}
\hline $\mathrm{I} 2-\mathrm{C} 2$ & $2.0916(16)$ & $\mathrm{C} 1-\mathrm{C} 7$ & $1.438(3)$ \\
$\mathrm{I} 4-\mathrm{C} 4$ & $2.094(2)$ & $\mathrm{C} 2-\mathrm{C} 3$ & $1.389(2)$ \\
$\mathrm{N} 7-\mathrm{C} 7$ & $1.151(3)$ & $\mathrm{C} 3-\mathrm{C} 4$ & $1.396(2)$ \\
$\mathrm{C} 1-\mathrm{C} 2$ & $1.405(2)$ & $\mathrm{C} 3-\mathrm{H} 3$ & 0.9500 \\
$\mathrm{C} 1-\mathrm{C} 2^{\mathrm{i}}$ & $1.405(2)$ & $\mathrm{C} 4-\mathrm{C} 3^{\mathrm{i}}$ & $1.396(2)$ \\
& & & 120.5 \\
$\mathrm{C} 2-\mathrm{C} 1-\mathrm{C} 2^{\mathrm{i}}$ & $119.9(2)$ & $\mathrm{C} 2-\mathrm{C} 3-\mathrm{H} 3$ & 120.5 \\
$\mathrm{C} 2-\mathrm{C} 1-\mathrm{C} 7$ & $120.07(11)$ & $\mathrm{C} 4-\mathrm{C} 3-\mathrm{H} 3$ & $121.6(2)$ \\
$\mathrm{C} 2-\mathrm{C} 1-\mathrm{C} 7$ & $120.07(11)$ & $\mathrm{C} 3-\mathrm{C} 4-\mathrm{C} 3$ & $119.19(11)$ \\
$\mathrm{C} 3-\mathrm{C} 2-\mathrm{C} 1$ & $120.19(16)$ & $\mathrm{C} 3-\mathrm{C} 4-\mathrm{I} 4$ & $119.19(11)$ \\
$\mathrm{C} 3-\mathrm{C} 2-\mathrm{I} 2$ & $120.65(12)$ & $\mathrm{C} 3-\mathrm{C} 4-\mathrm{I} 4$ &
\end{tabular}



$\mathrm{C} 1-\mathrm{C} 2-\mathrm{I} 2$
$119.16(13)$
$\mathrm{N} 7-\mathrm{C} 7-\mathrm{C} 1$
180.0
$\mathrm{C} 2-\mathrm{C} 3-\mathrm{C} 4$
$119.07(16)$
$\mathrm{C} 2 \mathrm{i}-\mathrm{C} 1-\mathrm{C} 2-\mathrm{C} 3$
$0.000(1)$
$180.000(1)$
$\mathrm{C} 7-\mathrm{C} 1-\mathrm{C} 2-\mathrm{C} 3$
$180.000(1)$
$\mathrm{C} 7-\mathrm{C} 1-\mathrm{C} 2-\mathrm{I} 2$
$0.000(1)$
$\mathrm{C} 1-\mathrm{C} 2-\mathrm{C} 3-\mathrm{C} 4$
$0.000(1)$
$\mathrm{I} 2-\mathrm{C} 2-\mathrm{C} 3-\mathrm{C} 4 \quad 180.000$ (1)
$\mathrm{C} 2-\mathrm{C} 3-\mathrm{C} 4-\mathrm{C}^{\mathrm{i}} \quad 0.000(1)$
$\mathrm{C} 2-\mathrm{C} 3-\mathrm{C} 4-\mathrm{I} 4 \quad 180.000$ (1)

Symmetry code: (i) $-x+1,-y+3 / 2, z$.

\section{2,4,6-Triiodophenyl isocyanide (I3NC)}

Crystal data

$\mathrm{C}_{7} \mathrm{H}_{2} \mathrm{I}_{3} \mathrm{~N}$

$M_{r}=480.80$

Orthorhombic, Imma

$a=7.0552(3) \AA$

$b=10.4947(5) \AA$

$c=13.1557$ (5) $\AA$

$V=974.08(7) \AA^{3}$

$Z=4$

$F(000)=840$

\section{Data collection}

Bruker VENTURE PHOTON-II

diffractometer

Radiation source: micro-focus

$\varphi$ and $\omega$ scans

Absorption correction: multi-scan

(SADABS; Sheldrick, 1996)

$T_{\text {min }}=0.251, T_{\max }=0.344$

13040 measured reflections

\section{Refinement}

Refinement on $F^{2}$

Least-squares matrix: full

$R\left[F^{2}>2 \sigma\left(F^{2}\right)\right]=0.011$

$w R\left(F^{2}\right)=0.024$

$S=1.14$

1314 reflections

40 parameters

0 restraints

Hydrogen site location: inferred from

neighbouring sites
$D_{\mathrm{x}}=3.279 \mathrm{Mg} \mathrm{m}^{-3}$

Melting point: $467 \mathrm{~K}$

Mo $K \alpha$ radiation, $\lambda=0.71073 \AA$

Cell parameters from 2833 reflections

$\theta=2.5-36.3^{\circ}$

$\mu=9.56 \mathrm{~mm}^{-1}$

$T=100 \mathrm{~K}$

Block, colourless

$0.14 \times 0.09 \times 0.07 \mathrm{~mm}$

1314 independent reflections 1267 reflections with $I>2 \sigma(I)$

$R_{\text {int }}=0.026$

$\theta_{\text {max }}=36.3^{\circ}, \theta_{\min }=2.5^{\circ}$

$h=-11 \rightarrow 11$

$k=-17 \rightarrow 12$

$l=-21 \rightarrow 21$

H-atom parameters constrained

$w=1 /\left[\sigma^{2}\left(F_{\mathrm{o}}^{2}\right)+(0.0044 P)^{2}+1.2499 P\right]$

where $P=\left(F_{\mathrm{o}}{ }^{2}+2 F_{\mathrm{c}}{ }^{2}\right) / 3$

$(\Delta / \sigma)_{\max }=0.001$

$\Delta \rho_{\max }=0.72 \mathrm{e} \AA^{-3}$

$\Delta \rho_{\min }=-0.48$ e $\AA^{-3}$

Extinction correction: SHELXL2014

(Sheldrick, 2015b),

$\mathrm{Fc}^{*}=\mathrm{kFc}\left[1+0.001 \mathrm{xFc}^{2} \lambda^{3} / \sin (2 \theta)\right]^{-1 / 4}$

Extinction coefficient: 0.00312 (10)

Special details

Geometry. All esds (except the esd in the dihedral angle between two 1.s. planes) are estimated using the full covariance matrix. The cell esds are taken into account individually in the estimation of esds in distances, angles and torsion angles; correlations between esds in cell parameters are only used when they are defined by crystal symmetry. An approximate (isotropic) treatment of cell esds is used for estimating esds involving 1.s. planes. 
Fractional atomic coordinates and isotropic or equivalent isotropic displacement parameters $\left(\AA^{2}\right)$

\begin{tabular}{lllll}
\hline & $x$ & $y$ & $z$ & $U_{\text {iso }} * / U_{\text {eq }}$ \\
\hline $\mathrm{I} 12$ & 0.5000 & $0.46223(2)$ & $0.83472(2)$ & $0.01190(3)$ \\
$\mathrm{I} 14$ & 0.5000 & 0.7500 & $0.43715(2)$ & $0.01245(4)$ \\
$\mathrm{N} 17$ & 0.5000 & 0.7500 & $0.91223(14)$ & $0.0120(3)$ \\
$\mathrm{C} 11$ & 0.5000 & 0.7500 & $0.80681(15)$ & $0.0098(3)$ \\
$\mathrm{C} 12$ & 0.5000 & $0.63389(13)$ & $0.75389(11)$ & $0.0102(2)$ \\
$\mathrm{C} 13$ & 0.5000 & $0.63411(14)$ & $0.64790(11)$ & $0.0112(2)$ \\
$\mathrm{H} 13$ & 0.5000 & 0.5561 & 0.6113 & $0.013^{*}$ \\
$\mathrm{C} 14$ & 0.5000 & 0.7500 & $0.59640(15)$ & $0.0108(3)$ \\
$\mathrm{C} 17$ & 0.5000 & 0.7500 & $1.00074(17)$ & $0.0150(4)$ \\
\hline
\end{tabular}

Atomic displacement parameters $\left(\AA^{2}\right)$

\begin{tabular}{lllllll}
\hline & $U^{11}$ & $U^{22}$ & $U^{33}$ & $U^{12}$ & $U^{13}$ & $U^{23}$ \\
\hline $\mathrm{I} 12$ & $0.01610(5)$ & $0.00910(4)$ & $0.01051(4)$ & 0.000 & 0.000 & $0.00184(3)$ \\
$\mathrm{I} 14$ & $0.01561(6)$ & $0.01413(6)$ & $0.00761(5)$ & 0.000 & 0.000 & 0.000 \\
$\mathrm{~N} 17$ & $0.0134(7)$ & $0.0125(7)$ & $0.0102(7)$ & 0.000 & 0.000 & 0.000 \\
C11 & $0.0106(8)$ & $0.0106(7)$ & $0.0081(7)$ & 0.000 & 0.000 & 0.000 \\
C12 & $0.0121(5)$ & $0.0086(5)$ & $0.0098(5)$ & 0.000 & 0.000 & $0.0012(4)$ \\
C13 & $0.0138(6)$ & $0.0096(5)$ & $0.0101(5)$ & 0.000 & 0.000 & $0.0000(4)$ \\
C14 & $0.0124(8)$ & $0.0116(8)$ & $0.0086(7)$ & 0.000 & 0.000 & 0.000 \\
C17 & $0.0174(9)$ & $0.0148(9)$ & $0.0127(8)$ & 0.000 & 0.000 & 0.000 \\
\hline
\end{tabular}

Geometric parameters $(\AA, \stackrel{\circ}{)})$

\begin{tabular}{llll}
\hline $\mathrm{I} 12-\mathrm{C} 12$ & $2.0920(14)$ & $\mathrm{C} 11-\mathrm{C} 12$ & $1.4035(17)$ \\
$\mathrm{I} 14-\mathrm{C} 14$ & $2.095(2)$ & $\mathrm{C} 12-\mathrm{C} 13$ & $1.394(2)$ \\
$\mathrm{N} 17-\mathrm{C} 17$ & $1.164(3)$ & $\mathrm{C} 13-\mathrm{C} 14$ & 0.9500 \\
$\mathrm{~N} 17-\mathrm{C} 11$ & $1.387(3)$ & $\mathrm{C} 13-\mathrm{H} 13$ & $1.3922(17)$ \\
$\mathrm{C} 11-\mathrm{C} 12^{\mathrm{i}}$ & $1.4035(17)$ & $\mathrm{C} 14-\mathrm{C} 13^{\mathrm{i}}$ & $119.21(14)$ \\
& & & 120.4 \\
$\mathrm{C} 17-\mathrm{N} 17-\mathrm{C} 11$ & 180.0 & $\mathrm{C} 14-\mathrm{C} 13-\mathrm{C} 12$ & 120.4 \\
$\mathrm{~N} 17-\mathrm{C} 11-\mathrm{C} 12^{\mathrm{i}}$ & $119.75(9)$ & $\mathrm{C} 14-\mathrm{C} 13-\mathrm{H} 13$ & $121.76(18)$ \\
$\mathrm{N} 17-\mathrm{C} 11-\mathrm{C} 12$ & $119.74(9)$ & $\mathrm{C} 12-\mathrm{C} 13-\mathrm{H} 13$ & $119.12(9)$ \\
$\mathrm{C} 12^{\mathrm{i}}-\mathrm{C} 11-\mathrm{C} 12$ & $120.51(18)$ & $\mathrm{C} 13-\mathrm{C} 14-\mathrm{C} 13$ & $119.12(9)$ \\
$\mathrm{C} 13-\mathrm{C} 12-\mathrm{C} 11$ & $119.65(13)$ & $\mathrm{C} 13-\mathrm{C} 14-\mathrm{I} 14$ & $\mathrm{C} 13-\mathrm{C} 14-\mathrm{I} 14$ \\
$\mathrm{C} 13-\mathrm{C} 12-\mathrm{I} 12$ & $120.65(10)$ & & $0.000(1)$ \\
$\mathrm{C} 11-\mathrm{C} 12-\mathrm{I} 12$ & $119.70(11)$ & & $180.000(1)$ \\
$\mathrm{N} 17-\mathrm{C} 11-\mathrm{C} 12-\mathrm{C} 13$ & $180.000(1)$ & $\mathrm{C} 11-\mathrm{C} 12-\mathrm{C} 13-\mathrm{C} 14$ & $0.000(1)$ \\
$\mathrm{C} 12^{\mathrm{i}}-\mathrm{C} 11-\mathrm{C} 12-\mathrm{C} 13$ & $0.000(1)$ & $\mathrm{I} 12-\mathrm{C} 12-\mathrm{C} 13-\mathrm{C} 14$ & $180.000(1)$ \\
$\mathrm{N} 17-\mathrm{C} 11-\mathrm{C} 12-\mathrm{I} 12$ & $0.000(1)$ & $\mathrm{C} 12-\mathrm{C} 13-\mathrm{C} 14-\mathrm{C} 13^{\mathrm{i}}$ & $\mathrm{C} 12-\mathrm{C} 13-\mathrm{C} 14-\mathrm{I} 14$ \\
$\mathrm{C} 12^{\mathrm{i}}-\mathrm{C} 11-\mathrm{C} 12-\mathrm{I} 12$ & $180.000(1)$ & &
\end{tabular}

Symmetry code: (i) $-x+1,-y+3 / 2, z$. 